\title{
DEMANDA DE MADEIRA PARA SERRARIAS E POTENCIAL DE INVESTIMENTO FLORES- TAL NA BACIA HIDROGRÁFICA DO RIO PARDO, RS, BRASIL
}

\author{
TIMBER DEMAND FOR SAWMILLS AND FOREST INVESTMENT POTENTIAL IN THE \\ RIO PARDO WATERSHED, RS, BRAZIL
}

\author{
Jorge Antonio de Farias ${ }^{1}$ Leonardo Job Biali ${ }^{2}$ Carline Andréa Welter ${ }^{3}$ Pábulo Diogo de Souza ${ }^{4}$ \\ Paulo Renato Schneider ${ }^{1}$
}

\begin{abstract}
RESUMO
O trabalho teve como objetivo geral avaliar a demanda de madeira para as serrarias no Vale do Rio Pardo e, ainda, analisar a viabilidade financeira de implantar uma base florestal que supra a capacidade ociosa de processamento dessa região. O estudo foi desenvolvido na área central do Rio Grande do Sul, abrangendo 13 municípios que compõem a Bacia Hidrográfica do Rio Pardo. Para a obtenção dos dados foram realizadas entrevistas semiestruturadas com proprietários de serrarias da região. Essas foram avaliadas quanto ao seu consumo e capacidade instalada, que, respectivamente, foram de $7.005 \mathrm{~m}^{3} \cdot \mathrm{mês}^{-1}$ e $12.330 \mathrm{~m}^{3} \cdot \mathrm{mês}^{-1}$, apresentando uma capacidade ociosa na ordem de $63.900 \mathrm{~m}^{3}$.ano-1. O abastecimento de $30.336 \mathrm{~m}^{3}$.ano-1 ocorre através de fornecedores de fora da região. Com isso, verificou-se a necessidade de 1.836 ha para suprir a demanda anual destas serrarias. A substituição da importação de matéria-prima e o incremento da capacidade ociosa seriam responsáveis por gerar 55 empregos nas atividades de produção florestal e 471 empregos na indústria. Conforme análise financeira pelo Método de Monte Carlo, a receita anual gerada pelo empreendimento seria na ordem de $\mathrm{R} \$ 1.756 .347,00$, correspondente a uma área de colheita estimada em 122,40 ha, distribuída nos municípios da região.
\end{abstract}

Palavras-chave: desenvolvimento regional; fomento florestal; políticas públicas; economia florestal.

\begin{abstract}
The general objective of this study is to evaluate the demand for timber from the sawmills and to analyze the financial feasibility of establishing a forest base that meets the idle processing capacity. The study was conducted in the central portion of Rio Grande do Sul state, comprising 13 municipalities that constitute the Rio Pardo watershed. Data collection was done through interviews with farmers and sawmill owners. The sawmills of the region were evaluated as to their timber consumption and installed capacity, respectively amounting to $7,005 \mathrm{~m}^{3} \cdot \mathrm{month}^{-1}$ and $12,330 \mathrm{~m}^{3} \cdot \mathrm{month}^{-1}$, with an idle capacity of approximately 63,900 $\mathrm{m}^{3}$.year-1. The supply of $30,336 \mathrm{~m}^{3}$.year ${ }^{-1}$ is carried out by suppliers from other regions. This shows the need for 1,836 hectares for meeting the annual demand. The replacement of raw material imports and a better use of the idle capacity would translate into the creation of 55 jobs in forest production activities and 471 jobs in the industries. According to the financial analysis by Monte Carlo Method, the annual income generated

1 Engenheiro Florestal, Dr., Professor do Departamento de Ciências Florestais, Centro de Ciências Rurais, Universidade Federal de Santa Maria, Av. Roraima, 1000, CEP 97105-900, Santa Maria (RS), Brasil. fariasufsm@ gmail.com / paulors@smail.ufsm.br

2 Engenheiro Florestal, Dr., Professor do Departamento de Engenharia Florestal, Faculdade de Tecnologia, Universidade de Brasília, Campus Universitário Darcy Ribeiro, CEP 70910-900, Brasília (DF), Brasil. ljbiali@ gmail.com

3 Engenheira Florestal, MSc., Doutoranda do Programa de Pós-graduação em Engenharia Florestal, Centro de Ciências Rurais, Universidade Federal de Santa Maria, Av. Roraima, 1000, CEP 97105-900, Santa Maria (RS), Brasil. carlinewelter@gmail.com

4 Engenheiro Florestal, Mestrando do Programa de Pós-graduação em Engenharia Florestal, Centro de Ciências Rurais, Universidade Federal de Santa Maria, Av. Roraima, 1000, CEP 97105-900, Santa Maria (RS), Brasil. pabulodiogo@gmail.com
\end{abstract}

Recebido para publicação em 14/12/2011 e aceito em 11/09/2017

Ci. Fl., v. 28, n. 4, out. - dez., 2018 
by the enterprise would reach approximately $\mathrm{R} \$ 1,756,347.00$, corresponding to a harvest area estimated at 122.40 hectares, across the municipalities of the region.

Keywords: regional development; forest promotion; public policies; forest economy.

\section{INTRODUÇÃO}

As florestas podem constituir-se em mola propulsora do desenvolvimento. No sul do Brasil, há várias regiões que podem ilustrar esse fato, por exemplo, os municípios de Lages e Caçador, em Santa Catarina, Caxias do Sul e Vacaria, no Rio Grande do Sul, e toda região metropolitana de Curitiba, no Paraná, que certamente devem sua importância econômica à riqueza obtida do extrativismo florestal. Segundo Koch e Correia (2010), a colonização da Região Sul teve nas áreas florestais nativas seu principal recurso, das quais obtinha alimentação, energia e material para construção, sobretudo das florestas de araucária.

No Rio Grande do Sul, Brena e Pereira (2006) deixam claro que o aproveitamento da madeira oriunda do desmatamento, especialmente da araucária, alavancou a instalação e o desenvolvimento do primeiro ciclo da cadeia produtiva de base florestal, contribuindo substancialmente para a consolidação da matriz econômica do estado.

A região do Vale do Rio Pardo - RS, colonizada por alemães, caracterizou-se por um modelo de ocupação fundiária, com base nas pequenas propriedades rurais. Por meio do plantio do tabaco, da conservação de florestas, do consumo de lenha para cura das folhas de tabaco e, também, da topografia que dificultou a implantação de lavouras extensivas (ETGES, 2001). Em função destas características, criou-se um conceito de que a atividade florestal se resumia à produção de lenha, principalmente, nas florestas de rápido crescimento, especialmente o gênero Eucalyptus spp., que teria melhor uso para essa finalidade.

A região do Vale do Rio Pardo tem ampla vocação florestal, na melhor acepção do termo, em função da logística, topografia, fertilidade e regime pluviométrico. O crescimento das cidades como Santa Cruz do Sul, Vera Cruz, Venâncio Aires e Sobradinho aumentou a demanda por produtos florestais, além do uso de energia, principalmente, para processamento em serrarias.

Um exemplo desta mudança de mercado são as florestas plantadas na região Centro-Serra do Vale, tendo como destaque Sobradinho. Nessa região, as florestas de Eucalyptus spp. com fins energéticos originalmente passaram a ser comercializadas para outros usos, como varas para a construção civil em Santa Maria, permitindo uma melhor remuneração quando comparado à venda de lenha. A produção de pallets e outras embalagens de madeira tornou a atividade florestal muito interessante, do ponto de vista econômico.

Uma das maiores vantagens do setor florestal madeireiro reside no fato de o mesmo possuir a capacidade de agregar maior valor ao produto final do que a maioria dos produtos agrícolas. Um produto agrícola chega ao consumidor em média $20 \%$ mais caro do que saiu do campo, enquanto que um produto madeireiro pode subir $150 \%$ do preço original (SILVA et al., 2002). Diante disto, o pequeno produtor florestal, ao buscar a obtenção de maior lucro com a madeira, necessita estar preparado para oferecer produtos com maior valor agregado (MATTOS; SILVA; MAGALHÃES, 2004). Dessa forma, este estudo avaliou a demanda de madeira para as serrarias na Bacia Hidrográfica do Rio Pardo e, ainda, analisou a viabilidade financeira de implantar uma base florestal que supra a capacidade ociosa de processamento.

\section{MATERIAIS E MÉTODOS}

O estudo foi desenvolvido em 13 municípios do Rio Grande do Sul, que compõem a área de atuação do Comitê de Gerenciamento da Bacia Hidrográfica do Rio Pardo. Esta Bacia aflui no Rio Jacuí, sendo integrante da Região Hidrográfica do Guaíba. A área de drenagem da Bacia do Pardo é de 3.636,79 km², com extensão de $115 \mathrm{~km}$ e a largura de $35 \mathrm{~km}$, representando 1,3\% da área do estado e 4,3\% da Região Hidrográfica do Guaíba (COMITÊ PARDO, 2016).

Foi elaborado um questionário semiestruturado para obter informações de demanda de madeira, capacidade instalada e de que forma as políticas públicas e os programas de fomento têm repercutido entre os empresários das serrarias. A pesquisa foi aplicada de forma direta, por meio de entrevista e na presença do pesquisador, nos anos de 2008 e 2009. Segundo dados obtidos junto à agência do Departamento de 
Biodiversidade da Secretaria do Ambiente e Desenvolvimento Sustentável (DBIO/SEMA) de Santa Cruz do Sul - RS, a região em estudo possui 75 serrarias, das quais 63 serrarias foram avaliadas na pesquisa, representando uma intensidade amostral de $84 \%$.

A partir do levantamento do consumo, origem da madeira e capacidade instalada das serrarias, foi analisado o potencial de investimento na região, visando implantar uma base florestal que atendesse ao deficit existente de madeira. Elaboraram-se cenários com o plantio de Eucalyptus grandis, espécie mais cultivada na região. Os dados para a simulação de crescimento e de produção foram obtidos com o uso do programa SisEucalipto, desenvolvido pela Empresa Brasileira de Pesquisa Agropecuária (Embrapa). Foram considerados dois cenários de produção, com horizonte de planejamento de 15 anos, um destinado à madeira para serraria, com dois desbastes (aos 5 e 10 anos) e o outro apenas considerando o uso para energia, com cortes rasos e reforma a cada 5 anos. Considerou-se um índice de sítio 40 e um espaço vital de $6 \mathrm{~m}^{2}$.

A rentabilidade econômica dos cenários foi analisada na condição determinística, na qual se pressupõe $100 \%$ de certeza quanto aos valores das variáveis, e na condição dinâmica, na qual se considera o risco, representado por distribuições de probabilidade de ocorrência. Na primeira condição, a taxa mínima de atratividade utilizada foi de 6,75\% ao ano, aquela aplicada aos projetos de financiamento florestal do Banco Nacional de Desenvolvimento Econômico e Social (BNDES). O valor da madeira destinada para energia foi de $\mathrm{R} \$ 20,00 / \mathrm{m}^{3}$, e o preço da madeira destinada ao consumo em serrarias foi de $\mathrm{R} \$ 66,00 / \mathrm{m}^{3}$, preços médios praticados no mercado regional. Os critérios de análise econômica utilizados estão apresentados na Tabela 1.

Realizou-se uma análise de risco pelo Método de Monte Carlo, que consiste em simular variáveis previamente selecionadas no fluxo de caixa e calcular os novos indicadores financeiros. O conjunto de dados resultante pode ser analisado estatisticamente e fornece informações que auxiliam em uma avaliação mais precisa de projetos (SIMIONI; HOEFLICH, 2006).

Para a aplicação do Método de Monte Carlo, seguiu-se o roteiro:

a) Definição das variáveis para simulação: levou-se em consideração a importância sobre o resultado econômico e a maior oscilação entre os tratamentos. Sendo utilizado, portanto, o custo de implantação, os preços pagos pela madeira, a produtividade e a taxa de juros.

b) Simulação das variáveis: foram realizadas dez mil simulações das variáveis selecionadas, utilizando-se a planilha eletrônica Microsoft Office Excel ${ }^{\circledR}$, de modo a obter dez mil fluxos de caixa diferentes.

c) Cálculo dos parâmetros financeiros para os dez mil fluxos de caixa: conforme Tabela 1.

d) Cálculo da média e desvio padrão amostral: a média representa o valor mais provável de ser encontrado e o desvio padrão representa o risco do investimento.

e) Confecção dos histogramas de frequência: estimou-se a probabilidade do valor do VPL de maior ocorrência. Em planilha eletrônica Microsoft Office Excel ${ }^{\circledR}$ organizaram-se os 10.000 VPLs estimados em classes, obtendo-se a frequência em cada classe e, a frequência acumulada.

TABELA 1: Critérios de análise econômica para avaliação dos projetos de Eucalyptus grandis, Hill ex Maiden.

TABLE 1: Economic analysis criteria for evaluation of the projects of Eucalyptus grandis, Hill ex Maiden.

\begin{tabular}{|c|c|}
\hline Critério & Fórmula \\
\hline $\begin{array}{l}\text { Valor presente líquido } \\
\text { (VPL) }\end{array}$ & $V P L=\sum_{j=0}^{n} R j(1+i)^{-j}-\sum_{j=0}^{n} C j(1+i)^{-j}$ \\
\hline $\begin{array}{l}\text { Razão benefício/custo } \\
(\mathrm{B} / \mathrm{C})\end{array}$ & $B / C=\frac{\sum_{j=0}^{n} R_{j}(1+i)^{-j}}{\sum_{j=0}^{n} C_{j}(1+i)^{-j}}$ \\
\hline $\begin{array}{l}\text { Taxa interna de retorno } \\
\text { (TIR) }\end{array}$ & $\sum_{j=0}^{n} R j(1+i)^{-j}-\sum_{j=0}^{n} C j(1+i)^{-j}=0$ \\
\hline
\end{tabular}


TABELA 1: Continuação...

TABLE 1: Continued...

\begin{tabular}{cc}
\hline Critério & Fórmula \\
\hline $\begin{array}{c}\text { Valor anual equivalente } \\
\text { (VAE) }\end{array}$ & $\operatorname{VAE}=\frac{\operatorname{VPL}\left[(1+\mathrm{i})^{\mathrm{t}}-1\right](1+\mathrm{i})^{\mathrm{nt}}}{(1+\mathrm{i})^{\mathrm{nt}}-1}$ \\
$\begin{array}{c}\text { Custo médio de produção } \\
(\mathrm{CMPr})\end{array}$ & $\mathrm{CMPr}=\frac{\sum_{\mathrm{j}=0}^{\mathrm{n}} \mathrm{CTj}}{\sum_{\mathrm{j}=0}^{\mathrm{n}} \mathrm{QTj}}$ \\
\hline
\end{tabular}

Em que: $\mathrm{Cj}=$ custo no final do ano $\mathrm{j} ; \mathrm{Rj}=$ receita no final do ano $\mathrm{j} ; \mathrm{i}=$ taxa de desconto; $\mathrm{n}=$ duração do projeto, em anos; $\mathrm{CT}$ = custo total atualizado; $\mathrm{QTj}$ = produção total equivalente; $\mathrm{t}$ = número de períodos de capitalização.

No Método de Monte Carlo, realizou-se a aleatorização de 30\% nas variáveis: custo de implantação, preço da madeira e produtividade. Além disso, trabalhou-se a taxa de juros sob duas condições: aleatorizada, variando de 1 a 15\% ao ano; e fixa, considerando uma taxa mínima de atratividade (TMA) de $20 \%$ ao ano.

\section{RESULTADOS E DISCUSSÃO}

\section{Demanda e perfil das serrarias}

Nas 63 serrarias avaliadas observou-se um consumo de $7.005 \mathrm{~m}^{3} \cdot \mathrm{mês}^{-1}$ de madeira bruta (Tabela 2). A capacidade instalada de processamento das serrarias foi de $12.330 \mathrm{~m}^{3} \cdot \mathrm{mês}^{-1}$, representando uma capacidade ociosa equivalente a 43,19\%. Em termos volumétricos, corresponde a $5.325 \mathrm{~m}^{3} \cdot \mathrm{mês}^{-1}$, totalizando um volume $63.900 \mathrm{~m}^{3} . \mathrm{ano}^{-1}$.

TABELA 2: Número de serrarias estabelecidas e com registro regular junto ao órgão florestal, nos municípios da Bacia Hidrográfica do Rio Pardo.

TABLE 2: $\quad$ Number of sawmills established and regular registration with the forestry agency, in the municipalities of the Rio Pardo Watershed.

\begin{tabular}{ccccc}
\hline \multirow{2}{*}{ Municípios } & $\begin{array}{c}\text { Número de serrarias Número de serrarias } \\
\text { registradas (un) }\end{array}$ & $\begin{array}{c}\text { Capacidade } \\
\text { pesquisadas (un) }\end{array}$ & Produção atual $\left(\mathrm{m}^{3}\right)$ \\
\hline Barros Cassal & 4 & 4 & 880 & 253 \\
Boqueirão do Leão & 7 & 7 & 1.570 & 1.070 \\
Candelária & 5 & 5 & 640 & 305 \\
Gramado Xavier & 1 & 1 & 250 & 250 \\
Herveiras & 1 & 1 & 200 & 100 \\
Lagoão & 1 & 1 & 80 & 60 \\
Passa Sete & 2 & 2 & 400 & 206 \\
Rio Pardo & 3 & 0 & 0 & 0 \\
Santa Cruz do Sul & 20 & 17 & 3.360 & 2.420 \\
Sinimbu & 5 & 3 & 590 & 250 \\
Vale do Sol & 6 & 6 & 1.100 & 385 \\
Venâncio Aires & 19 & 10 & 2.400 & 1.435 \\
Vera Cruz & 6 & 6 & 860 & 271 \\
\hline Total & 75 & 63 & 12.330 & 7.005 \\
\hline
\end{tabular}


Estes resultados apontam o potencial instalado da região para ampliar a produção de serrados. Políticas públicas visando maximizar o uso deste recurso industrial trariam um efeito direto na geração de emprego e renda. Mas, para isso, é necessário garantir o suprimento de madeira de qualidade e dimensões apropriadas. A ideia recorrente de que o melhor investimento em florestas é para produção de lenha e, recentemente, a pressão sobre as florestas de eucaliptos, pelas serrarias dedicadas à produção de pallets, não tem permitido que florestas de eucalipto alcancem rotações mais longas.

As serrarias, localizadas na área de estudo, têm idade média de 25,3 anos, existindo empresas com menos de 1 ano de atividade e outras em torno de 80 anos, que se encontram na segunda ou terceira geração de administração. A fonte principal de madeira das serrarias são florestas do gênero Eucalyptus $(84,6 \%)$, do gênero Pinus sp. $(15,1 \%)$ e outras espécies $(0,3 \%)$.

$\mathrm{O}$ valor pago pelas serrarias aos fornecedores é praticamente idêntico para os gêneros Pinus e Eucalyptus (entre R\$ 65,00 e R\$ 70,00 por metro cúbico em pé). Os valores são atrativos, já que, além do valor em si, o produtor fica com todos os galhos e a ponta da árvore para consumo ou para comercialização como lenha.

Dos fornecedores, $85,5 \%$ são da própria região, entretanto, respondem por $63,9 \%$ do volume ofertado. Isso faz com que mais de $1 / 3$ da madeira consumida necessite ser importada de outras regiões. Considerando o consumo de $7.005 \mathrm{~m}^{3}$.mês ${ }^{-1}$, há uma evasão de divisas na ordem de R $\$ 167.407,90$ mês $^{-1}$.

Os proprietários das serrarias têm um elevado grau de conhecimento em relação à legislação do setor, especificamente a legislação pertinente à silvicultura, não tendo sido feitas perguntas que envolvessem a legislação tributária, trabalhista, etc. A grande maioria das serrarias não sofreu autuação pelos órgãos ambientais e seus agentes, como também demonstram que há um pleno conhecimento sobre o conteúdo desta legislação de maneira que, na avaliação dos entrevistados, não representa um problema significativo (Figura 1).

Naturalmente, boa parte deste cenário, explica-se pelo uso integral de madeira oriunda de florestas plantadas. No caso dos empreendedores que têm interesse em plantios florestais com espécies nativas, é fundamental que tenham as mesmas garantias legais que são dadas às espécies exóticas, para que haja segurança de uso no futuro. Caso contrário, as opções se restringirão unicamente às espécies exóticas.

A Figura 2 mostra a percepção que os proprietários de serraria têm das políticas públicas relacionadas à atividade florestal. Observou-se que a maioria dos empresários não participa de cursos e treinamento sobre o seu negócio. Talvez, porque não tenha surgido oportunidade ou porque a pergunta não foi bem específica se participaria ou não. Por outro lado, percebe-se que a maioria participa de palestras sobre florestamento, confirmando a hipótese da oportunidade.

Foi possível notar que estes empreendedores têm informação sobre o PRONAF Florestal, programa de financiamento com o perfil dos seus fornecedores. Quando lhes foi perguntado sobre o PROPFLORA, a esmagadora maioria não tinha conhecimento do que se trata e, ao mesmo tempo, revelou interesse em fazer plantios florestais próprios. Então, constatou-se que, claramente, as políticas públicas não estão atingindo seus objetivos.

Os programas de financiamentos que podem auxiliar as serrarias são desconhecidos pelos empresários, seja na forma de modernização das máquinas ou obtenção de capital de giro. Isso revela uma falha na formulação das políticas, cuja característica é a segmentação dos programas, ao invés de constituir um programa que possa implementar a cadeia produtiva como um todo. Há necessidade de criar programas não somente para viabilizar financeiramente os empreendimentos, mas sim, também para dar suporte tecnológico em todos os segmentos da cadeia. 


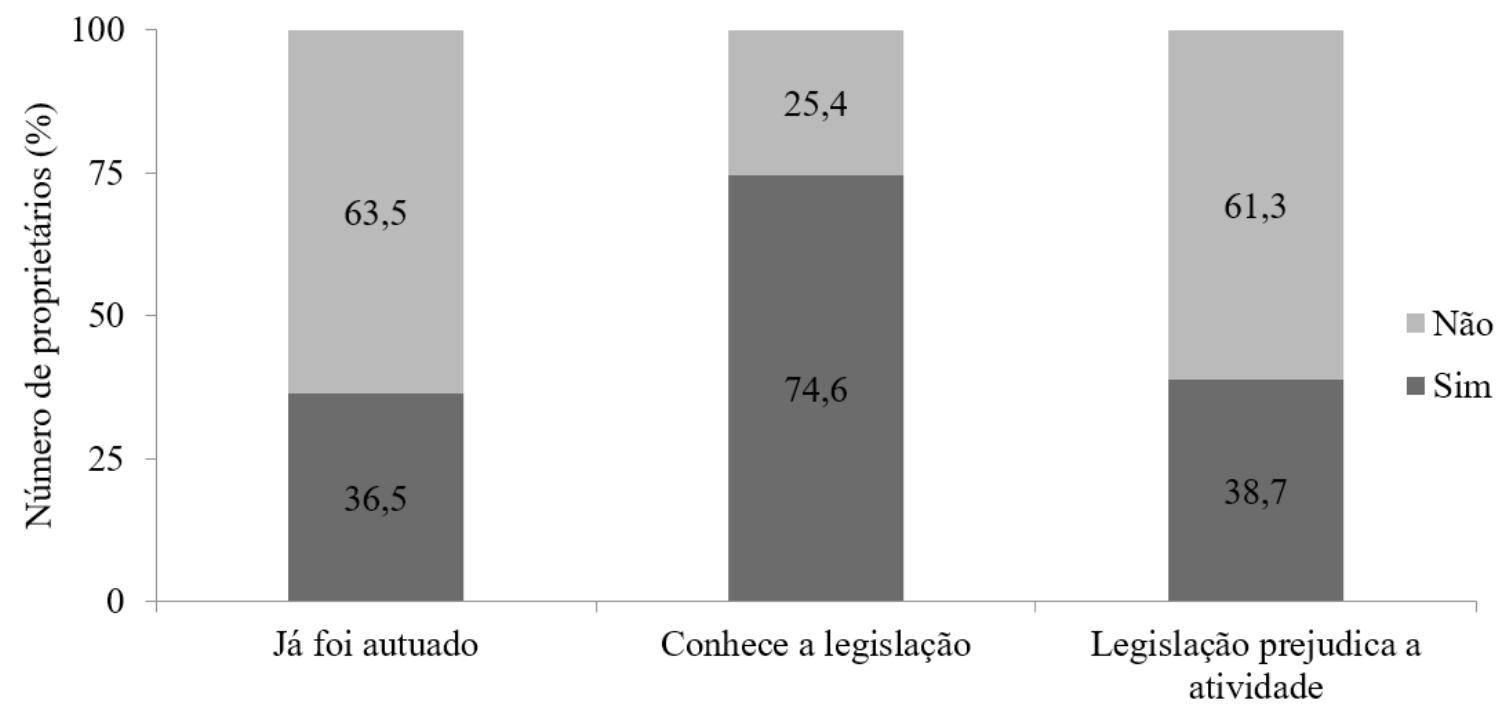

FIGURA 1: Percepção dos proprietários de serrarias, relacionando sua atividade com a legislação. FIGURE 1: Perception of the owners of sawmills, relating its activity with the legislation.

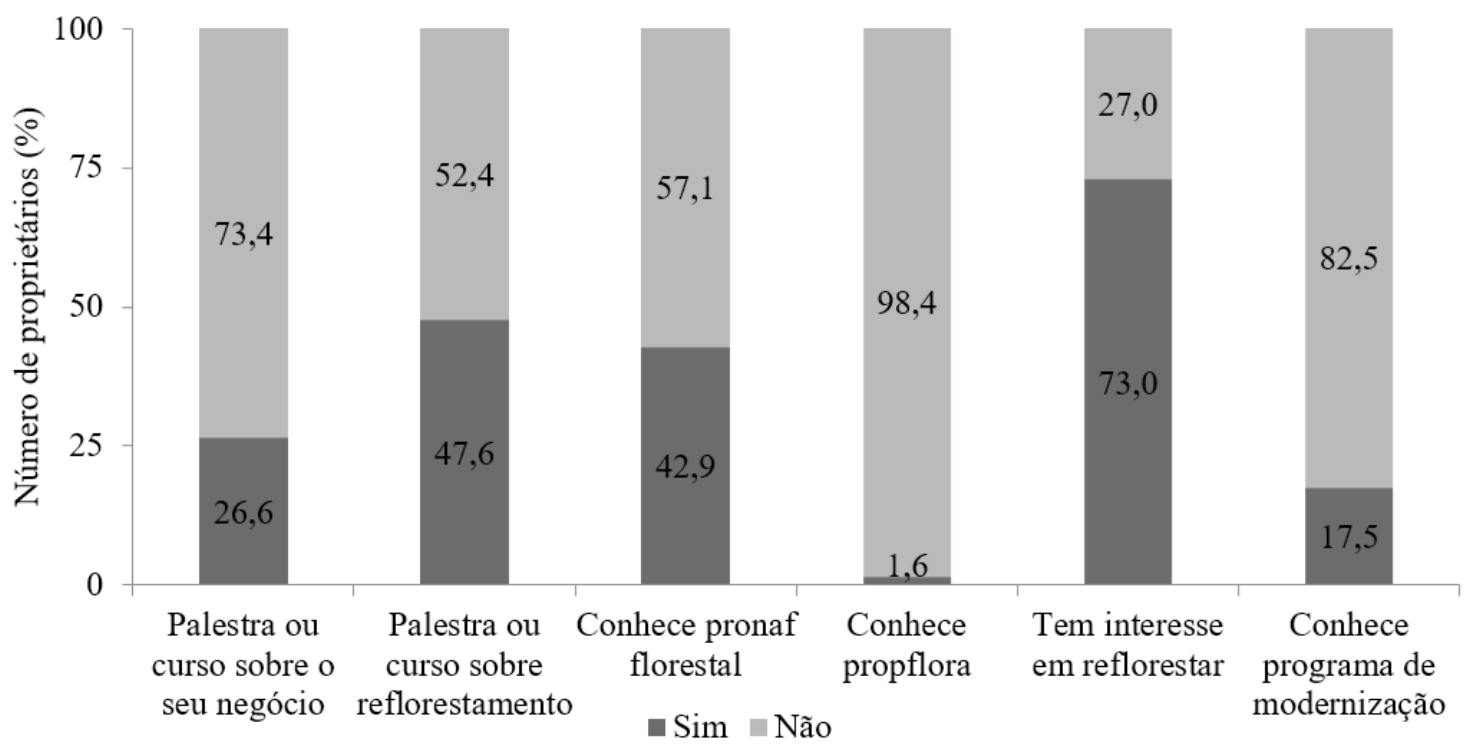

FIGURA 2: Nível de informação dos proprietários de serraria em relação às políticas públicas e programas de modernização.

FIGURE 2: Level of information of the sawmill owners in relation to public policies and programs of modernization.

Este gargalo pode ser observado nos dados da Figura 3, na qual se percebe que há procura por madeira serrada seca, mas a maioria não tem estrutura para secar a madeira. Fica evidente que caberia um programa de modernização do parque industrial, não somente em relação à secagem de madeiras, mas uma atualização dos equipamentos como um todo, que apresentam baixas produtividades e rendimento operacional inferior a $50 \%$.

Os empresários estão dispostos a remunerar melhor a tora desramada. Mas para isso, há necessidade de aprimorar além das serrarias, toda cadeia de suprimentos. Sendo fornecida assistência técnica competente, que possa orientar os produtores rurais, acerca das práticas corretas de desrama. Isso traria um efeito 
catalisador para todo o setor pela possibilidade de agregação de valor, o que pode ser comprovado pelos valores pagos para cada segmento de mercado, sendo madeira bruta para o atacado (R\$296,20), madeira bruta para o beneficiamento ( $\mathrm{R} \$ 380,00)$ e madeira beneficiada ( $\mathrm{R} \$ 435,20)$, em que a última consegue uma remuneração $46,9 \%$ superior.

Os empresários acreditam na atividade, tanto que a maioria possui florestamentos (58,7\%). Entretanto, ainda muito longe de atingir autossuficiência, apenas 2,2\% das serrarias trabalham exclusivamente com madeira própria. $\mathrm{O}$ que garante segurança aos produtores rurais que optarem por efetuar plantios florestais, para abastecer o mercado.

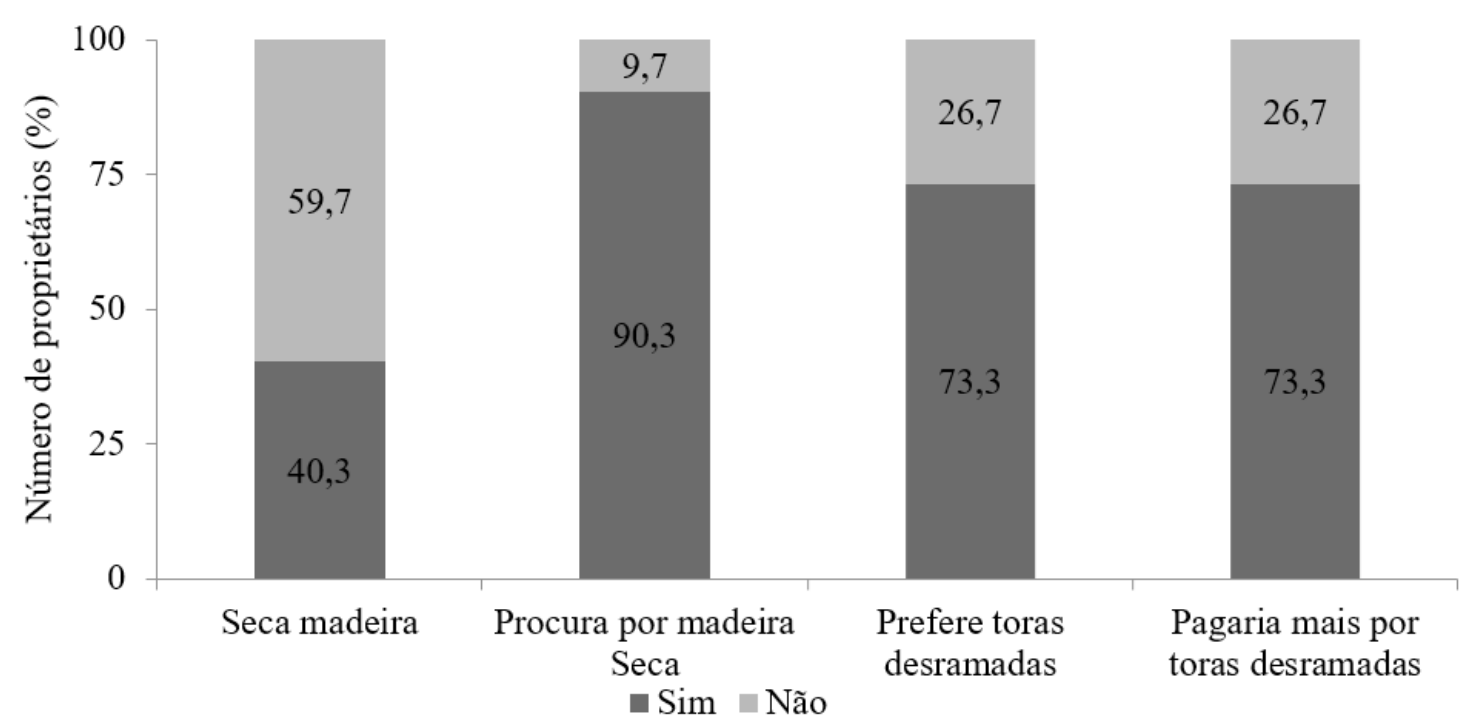

FIGURA 3: Oportunidade de ampliar negócios com madeira desramada e seca.

FIGURE 3: Opportunity to expand business with pruned and dry wood.

Isso demonstra a capacidade que as estruturas, já existentes do setor de serrarias da região, possuem como alternativa para as famílias rurais, uma vez que há espaço para ampliar os negócios, visando gerar mais renda principalmente no contexto da agricultura familiar.

Para isso, há que se discutir os investimentos necessários neste processo, não somente econômicos, mas em capacitação, profissionalização e infraestrutura. Da mesma forma, é importante a descentralização dos investimentos, fortalecendo as pequenas serrarias localizadas no interior, porque são essas os principais consumidores de madeira das florestas produzidas na pequena propriedade.

A estruturação da cadeia produtiva na agricultura familiar e não uma indústria-âncora é que permite estabelecer um processo de desenvolvimento regional mais harmonioso, evitando a concentração de áreas e renda. Neste caso, ao contrário das propostas correntes que estabelecem como peça-chave a indústria de transformação, a proposta de uma cadeia produtiva florestal para a agricultura familiar é de que o elemento social seja considerado, ou seja, a fonte da matéria-prima é que deve ser a base da cadeia.

\section{Potencial de investimento na região}

A região em estudo apresenta uma capacidade ociosa nas serrarias na ordem de $63.900 \mathrm{~m}^{3}$.ano- ${ }^{-1}$. Também apresenta um abastecimento através de fornecedores de fora da região de $30.336 \mathrm{~m}^{3}$.anototalizando um deficit de $94.236 \mathrm{~m}^{3}$.ano-1. Considerando um incremento médio anual (IMA) de 51,34 $\mathrm{m}^{3} \cdot \mathrm{ha}^{-1}$.ano (FARIAS, 2010), seriam necessários 1.836 ha para suprir a demanda anual.

Para Schneider (2005), o setor de madeira serrada pode gerar 3 empregos diretos para cada 100 ha florestados e 5 empregos para cada $1.000 \mathrm{~m}^{3}$.ano ${ }^{-1}$, processados pelas empresas. Dessa forma, o número de empregos gerados, através da substituição da importação de matéria-prima e incremento da capacidade 
ociosa, seria responsável por gerar 55 empregos nas atividades de produção florestal e 471 empregos na indústria.

Na Tabela 3 estão os valores dos investimentos necessários para este empreendimento em área de eucalipto plantada, enquanto na Tabela 4 são apresentados os valores da análise financeira determinística, considerando uma rotação de 15 anos.

Os dados de produtividade considerados, conforme SisEucalipto, foram:

- Cenário 1 (alto fuste): aos 5 anos, 70,5 $\mathrm{m}^{3} \cdot \mathrm{ha}^{-1}$ de madeira para energia; aos 10 anos, $121,1 \mathrm{~m}^{3} \cdot \mathrm{ha}^{-1}$ para energia e $139,8 \mathrm{~m}^{3} \cdot \mathrm{ha}^{-1}$ de madeira para serraria; aos 15 anos, $91,3 \mathrm{~m}^{3} \cdot \mathrm{ha}^{-1}$ para energia e $347,4 \mathrm{~m}^{3} \cdot \mathrm{ha}^{-1}$ para serraria.

- Cenário 2 (energia): 412,6 $\mathrm{m}^{3} \cdot \mathrm{ha}^{-1}$ a cada 5 anos.

TABELA 3: Volume de recursos necessários para implantação do projeto florestal para uma área de 1.836 hectares, com espaço vital de $6 \mathrm{~m}^{2}$.

TABLE 3: Volume of resources necessary needed to implement the project for a forest area of 1.836 hectares, with $6 \mathrm{~m}^{2}$ of living space.

\begin{tabular}{ccccc}
\hline Insumo & Quantidade (und) & Valor unitário (R\$) & Valor total (R\$) & Valor (R\$.ha $\left.{ }^{-1}\right)$ \\
\hline Mudas de Eucalipto (muda) & 3.121 .200 & 0,18 & $561.816,00$ & 306 \\
Formicida (kg) & 5.508 & 9,35 & $51.499,80$ & 28,05 \\
Fertilizantes (sc) & 6.242 & 83 & $518.119,20$ & 282,2 \\
Herbicidas (l) $_{\text {Mão de obra }}$ (ha) & 6.885 & 27 & $185.895,00$ & 101,25 \\
Preparo de solo (ha) $_{\text {Total }}^{1.836}$ & 700 & $1.285 .200,00$ & 700 \\
\hline & 1.836 & 250 & $459.000,00$ & 250 \\
\hline
\end{tabular}

'A mão de obra considerada é apenas familiar, com a contratação eventual de terceiros, os serviços de preparo de solo são na maioria das vezes com tração animal

TABELA 4: Indicadores econômicos pela análise determinística.

TABLE 4: Economic indicators of the deterministic analysis.

\begin{tabular}{ccc}
\hline Indicadores Econômicos & Cenário 1 (alto fuste) & Cenário 2 (energia) \\
\hline Valor Presente Líquido $\left(\mathrm{R} \$ . \mathrm{ha}^{-1}\right)$ & $14.703,98$ & $9.606,55$ \\
Taxa Interna de Retorno $\left(\% . \mathrm{ano}^{-1}\right)$ & 30,31 & 37,69 \\
Relação Benefício/Custos & 9,82 & 3,57 \\
Valor Equivalente Anual $\left(\mathrm{R} \$ . \mathrm{ha}^{-1} . \mathrm{ano}\right)$ & $1.589,02$ & $1.038,15$ \\
Custo Médio de Produção $\left(\mathrm{R} \$ . \mathrm{m}^{-3}\right)$ & 4,75 & 10,64 \\
\hline
\end{tabular}

Os resultados obtidos com o Método de Simulação de Monte Carlo são apresentados na Tabela 5, e indicam uma alta viabilidade dos projetos. Após a construção das distribuições de frequência dos fluxos de caixa via Método de Monte Carlo (10.000 simulações), foi possível a construção de histogramas (Figura 4).

Observou-se que, apenas no cenário para energia com TMA de $20 \%$, houve inviabilidades financeiras, com valores negativos de VPL e VAE, porém, ocorrentes em menos de 1\% dos casos da simulação. Ainda, apenas nesse panorama, o retorno financeiro seria superior ao cenário de alto fuste, demonstrando que a condução do reflorestamento para a geração de produtos de maiores diâmetros destinada às serrarias é uma boa alternativa econômica para a agricultura familiar da região. 
TABELA 5: Indicadores econômicos, considerando-se análise de risco (Método de Monte Carlo) para a avaliação dos projetos de Eucalyptus grandis, Hill ex Maiden.

TABLE 5: Economic indicators, considering risk analysis (Monte Carlo Method) for the evaluation of projects of Eucalyptus grandis, Hill ex Maiden.

\begin{tabular}{|c|c|c|c|c|c|c|c|c|c|}
\hline \multirow{2}{*}{ Cenários } & \multirow{2}{*}{ Critérios } & \multicolumn{4}{|c|}{ Alto fuste } & \multicolumn{3}{|c|}{ Energia } & \multirow[b]{2}{*}{ Desvio } \\
\hline & & Média & Máximo & Mínimo & Desvio & Média & Máximo & Mínimo & \\
\hline \multirow[t]{5}{*}{ TMA $20 \%$ a.a. } & VPL & $2.386,68$ & $4.700,32$ & 228,95 & 727,55 & $2.585,82$ & $6.836,78$ & $-677,98$ & $1.360,54$ \\
\hline & TIR & $30,40 \%$ & $40,26 \%$ & $21,15 \%$ & $3,01 \%$ & $37,51 \%$ & $63,77 \%$ & $14,63 \%$ & $8,43 \%$ \\
\hline & VAE & 510,47 & $1.005,31$ & 48,97 & 155,61 & 553,06 & $1.462,27$ & $-145,01$ & 290,99 \\
\hline & $\mathrm{B} / \mathrm{C}$ & 2,51 & 5,01 & 1,11 & 0,61 & 2,29 & 5,28 & 0,89 & 0,71 \\
\hline & CMPr & 16,99 & 28,44 & 9,9 & 3,26 & 9,32 & 16,68 & 4,88 & 2,34 \\
\hline \multirow{5}{*}{$\begin{array}{l}\text { Taxa aleatorizada } \\
1 \mathrm{a} \quad 15 \% \text { a.a. }\end{array}$} & VPL & $14.349,24$ & 47.741, & $2.404,0$ & $8.156,18$ & $9.324,30$ & $33.184,22$ & 31,82 & $5.143,44$ \\
\hline & TIR & $30,42 \%$ & $39,85 \%$ & $20,90 \%$ & $3,06 \%$ & $37,53 \%$ & $63,41 \%$ & $13,78 \%$ & $8,43 \%$ \\
\hline & VAE & $1.496,29$ & $3.459,96$ & 404,61 & 524,18 & 995,35 & $2.434,07$ & 5,2 & 392,34 \\
\hline & $\mathrm{B} / \mathrm{C}$ & 9,91 & 37,32 & 2,14 & 5,45 & 4,65 & 15,82 & 1,19 & 1,91 \\
\hline & $\mathrm{CMPr}$ & 5,91 & 15,98 & 1,55 & 2,75 & 6,2 & 14,35 & 2,37 & 1,97 \\
\hline
\end{tabular}

Quando considerada uma variação da taxa de juros, o cenário de alto fuste se mostrou o mais consistente, não apresentando nas simulações situação de inviabilidade financeira. Os valores médios de VPL, TIR, VAE, B/C, CMPr foram, respectivamente de R\$ 14.349,24 ha ${ }^{-1}, 30,42 \%$, R $\$ 1.496,29$ ha $^{-1}$.ano, 9,91, $\mathrm{R} \$ 5,91 \mathrm{~m}^{-3}$.

Considerando-se que a área deste projeto compreende 1.836 ha, a receita anual gerada pelo empreendimento será na ordem de R $\$ 1.756 .346,98$, correspondente a uma área de colheita estimada em 122,40 ha. Desse modo, o uso dos indicadores econômicos combinados às simulações do Método de Monte Carlo evidencia a viabilidade dos cenários. Contudo, deve-se observar como as variáveis irão se comportar para que seja possível uma análise apurada de probabilidade de ocorrência destes, pois consideram os riscos associados. 


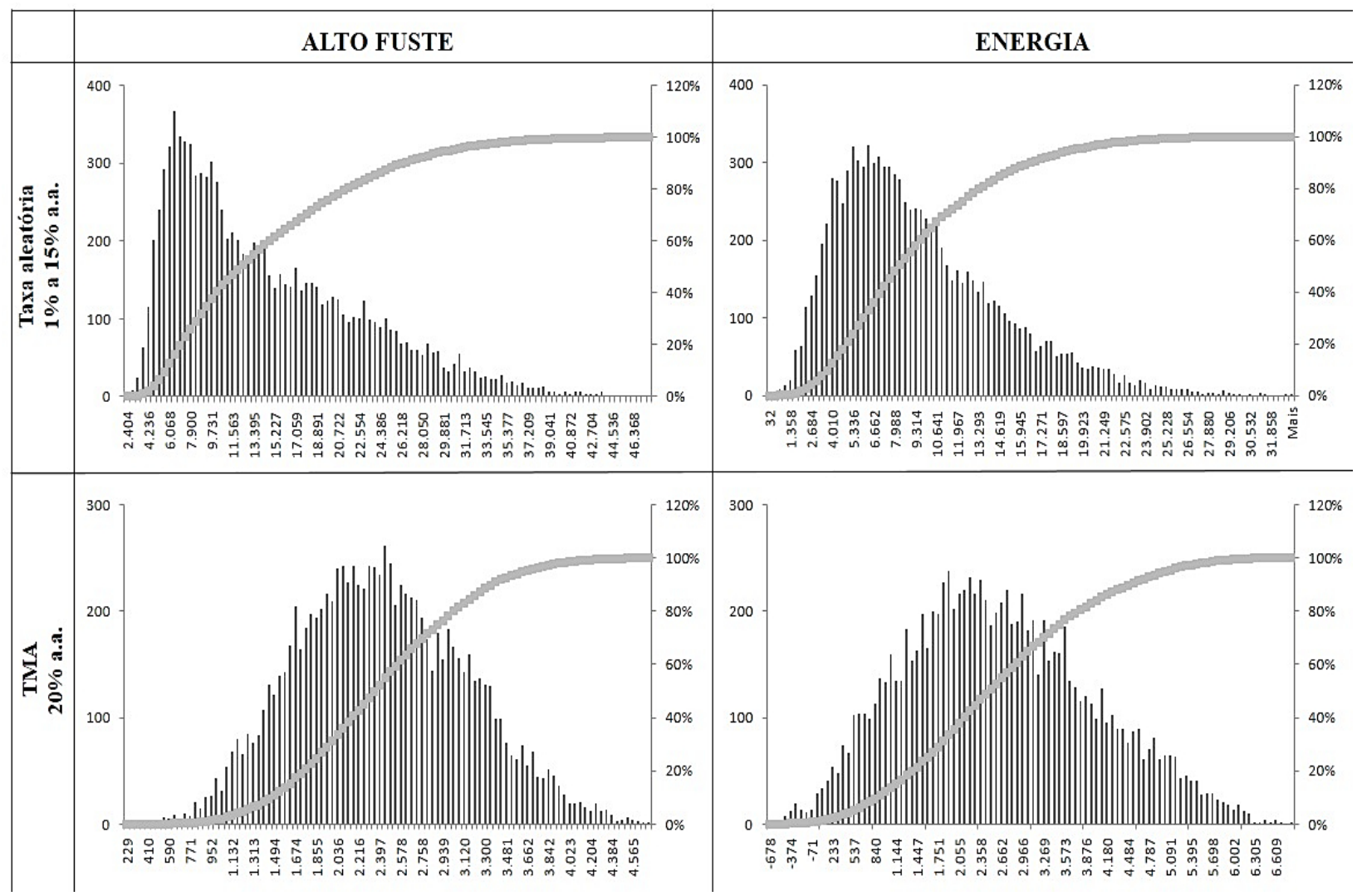

FIGURA 4: Histograma de frequências e curva de distribuição de frequência acumulada resultantes do Método de Monte Carlo para o valor presente líquido (VPL) dos projetos de investimento em florestamento com Eucalyptus grandis Hill ex Maiden.

FIGURE 4: Histogram of frequency and cumulative frequency distribution curve resulting from the Monte Carlo Method for the net present value (NPV), investment projects on forestation with Eucalyptus grandis Hill ex Maiden.

\section{CONCLUSÕES}

Os resultados obtidos permitiram concluir, para a área de abrangência do presente estudo, que: 1) $\mathrm{O}$ volume de madeira processada pelas serrarias ocupa pouco mais da metade da capacidade instalada; $\mathrm{e}$ da demanda observada, mais de 1/3 é oriunda de outras regiões;

2) Há um mercado potencial para produção e comercialização de toras de qualidade, já que o deficit regional é superior a $50 \%$ da capacidade de processamento e, ainda, as serrarias se mostram dispostas a pagar mais pela madeira desramada;

3) Os proprietários das serrarias demonstram conhecimento sobre a legislação relacionada aos florestamentos e possuem interesse em investir em plantios. Por outro lado, desconhecem as principais linhas de financiamento para a atividade;

4) A análise financeira tanto na condição determinística quanto dinâmica (Método de Monte Carlo) indicou a viabilidade de implantação de uma base florestal que atenda à demanda de madeira das serrarias, com um retorno superior ao observado para florestamentos direcionados para produzir biomassa em ciclos curtos de rotação. 


\section{REFERÊNCIAS}

BRENA, D. A.; PEREIRA, O. S. Programa para o desenvolvimento sustentável da cadeia produtiva de base florestal no Rio Grande do Sul - Período 2007 - 2027. Santa Maria: UFSM, 2006.

COMITÊ PARDO. Síntese da paisagem da Bacia. [2016]. Disponível em: <http://www.comitepardo.com. br/mapas/01_unidades_estudo_malha_municipal.htm>. Acesso em: 04 jun. 2016.

ETGES, V. E. A região no contexto da globalização: o caso do Vale do Rio Pardo. In: VOGT, O. P.; SILVEIRA, R. L. (Org.). Vale do Rio Pardo: (re)conhecendo a região. Santa Cruz do Sul: Edunisc, 2001. p. 351-366.

FARIAS, J. A. Atividade florestal no contexto da fumicultura: oportunidade de desenvolvimento regional, diversificação, geração de emprego e renda. 2010. 166 f. Tese (Doutorado em Engenharia Florestal) Universidade Federal de Santa Maria, Santa Maria, 2010.

KOCH, Z:; CORRÊA, M. C. Araucária: a floresta do Brasil Meridional. 2. ed. Rio de Janeiro: Olhar Brasileiro, 2010. 148 p.

MATTOS, P. P.; SILVA, V. P.; MAGALHÃES, W. L. E. Agregação de valor à pequena produção florestal madeireira. Colombo: Embrapa Floresta, 2004.

SCHNEIDER, P. R. et al. Plano estratégico de desenvolvimento florestal: região central do Rio Grande do Sul. Santa Maria: FACOS; UFSM, 2005. 210 p.

SILVA, H. D.; BELLOTE, A. F. J.; FERREIRA, C. A. Cultivo do eucalipto. Colombo: Embrapa Florestas, 2002.

SIMIONI, F. J.; HOEFLICH, V. A. Avaliação de risco em investimentos florestais. Boletim de Pesquisa Florestal, Colombo, n. 52, p. 79-92, 2006. 